\title{
Long-Term Progression-Free Survival in Anaplastic Lymphoma Kinase-Positive Non-Small Cell Lung Cancer Patient Treated With Crizotinib
}

\author{
Emad Mohsen Barsoum ${ }^{\mathrm{a}}$, Mahmoud Mohammed Eldemery ${ }^{\mathrm{b}}$, Nada Osama Osman ${ }^{\mathrm{c}}$, \\ May Gamal Ashour ${ }^{\text {d, e }}$, Mohsen Samy Barsoum ${ }^{\mathrm{a}}$
}

\begin{abstract}
Lung cancer is the second most common cancer worldwide. Nonsmall cell lung cancer (NSCLC) with anaplastic lymphoma kinase $(A L K)$ gene rearrangements constitutes 3-5\%. Crizotinib was approved for the first-line therapy of advanced ALK-positive NSCLC patients. We present a female patient with advanced ALK-positive NSCLC who was kept on crizotinib as first-line therapy and showed progression-free survival (PFS) of 48 months despite the data suggesting that the majority of patients on crizotinib show relapse within 1 year. Further studies should focus on the molecular and biological factors and the possible effect of the long-term use of this drug.
\end{abstract}

Keywords: Crizotinib; $A L K$; Tyrosine kinase inhibitors; Non-small cell lung cancer

\section{Introduction}

Rearrangements of the anaplastic lymphoma kinase $(A L K)$ gene occur in $3-5 \%$ of patients with non-small cell lung cancer (NSCLC) [1]. In addition, it is enriched in patients with adenocarcinoma histology, young age, and never or light smokers [2].

There are multiple ALK tyrosine kinase inhibitors (TKIs), such as crizotinib, ceritinib, alectinib, brigatinib, and lorlat-

Manuscript submitted July 6, 2021, accepted August 2, 2021

Published online August 25, 2021

aBarsoum Oncology Center (BOC), Cairo, Egypt

${ }^{\mathrm{b}}$ Ahmed Maher Teaching Hospitals, Cairo, Egypt

'Kasr Al-Ainy Center of Clinical Oncology and Nuclear Medicine, Kasr AlAiny School of Medicine, Cairo University, Cairo, Egypt

${ }^{\mathrm{d}}$ Radiation Oncology and Nuclear Medicine Department, National Cancer Institute, Cairo University, Cairo, Egypt

${ }^{\mathrm{e} C o r r e s p o n d i n g ~ A u t h o r: ~ M a y ~ G a m a l ~ A s h o u r, ~ R a d i a t i o n ~ O n c o l o g y ~ a n d ~ N u c l e a r ~}$ Medicine Department, National Cancer Institute, Cairo University, Cairo,

Egypt.Email: may.ashour@nci.cu.edu.eg

doi: https://doi.org/10.14740/jmc3743 inib, developed for ALK-positive NSCLC [3].

Crizotinib (Xalkori) was the first approved potent inhibitor for ALK, mesenchymal-epithelial transition, and ROS1 tyrosine kinase receptor [4]. In addition, it has more prolonged progression-free survival (PFS), higher objective response rate, and better patient-reported outcomes $[5,6]$.

\section{Case Report}

\section{Investigations}

A 38-year-old female patient, a non-smoker, presented with hemoptysis and right-sided chest pain.

Chest computed tomography (CT) showed an ill-defined patchy area of pulmonary opacity in the right lung associated with right pleural effusion.

Positron emission tomography/CT (PET/CT) showed low-grade metabolically active middle lobe consolidative lesion (maximum standardized uptake value (SUV max) 3.3), multiple right pleuro-pulmonary nodules (SUV max 2.1), right pleural effusion and hypermetabolic small mediastinal nodes (SUV max 4.8). Bronchoscopic biopsy and bronchoalveolar lavage cytology were done.

\section{Diagnosis}

The results are positive for bronchogenic adenocarcinoma carcinoma, grade 2, and confirmed with immunophenotyping. Epidermal growth factor receptor (EGFR) test revealed no mutations by liquid biopsy (from malignant effusion). The adenocarcinoma is positive for $A L K$ gene rearrangement. $A L K$ gene was tested by fluorescent in situ hybridization (FISH) and polymerase chain reaction (PCR). The patient's clinical stage was T4N2M1a (stage IV).

\section{Treatment}

Our patient started treatment with crizotinib $250 \mathrm{mg}$ twice daily in February 2017. 


\section{Follow-up and outcomes}

Follow-up CT after 4 months showed a regressive course of the disease. On clinical examination, we noticed a mild degree of bilateral lower limb edema; lab results were all within normal range except high liver enzymes (alanine aminotransferase (ALT) and aspartate transaminase (AST), total bilirubin $2.9 \mathrm{mg} / \mathrm{dL}$, direct bilirubin $1.1 \mathrm{mg} / \mathrm{dL}$ ), which were normalized after modifying her diet and adding ursodeoxycholic acid for 2 weeks. Thus, the patient was reassured and advised to continue treatment.

She continued on crizotinib with a regressive disease course until August 2018; PET/CT showed complete resolution of lung and nodal disease, but the patient complained of severe pain at the left femur. Magnetic resonance imaging (MRI) hip joints revealed a small area of marrow edema signal at the left femoral head; lab results were normal except for vitamin D deficiency (25-hydroxyl vitamin D was $7.36 \mathrm{ng} / \mathrm{mL}$ (reference range: $30-100)$ ). She was referred to an orthopedic surgery consultant. One month later, her femur was broken, underwent surgical fixation; excision was done, showing avascular necrosis with evidence of stress fracture and no malignancy.

She had regular follow-ups with the orthopedist, and continued on crizotinib. A complete response was maintained till the time of publication.

\section{Discussion}

Lung cancer is the second most common cancer worldwide, and is the leading cause of cancer death in men and women, accounting for over $25 \%$ of all cancer fatalities [7]. NSCLC presents $85 \%$ of all lung cancer cases [8]. Paradigm-changing studies have reshaped the concept of NSCLC from being a single disease or histology-based subgroups to the concept of a large number of molecularly distinct subtypes.

The last decade has witnessed the emergence of molecularly targeted therapy to treat ALK-positive lung cancer. Crizotinib (first-generation ALK inhibitor) was approved by the US Food and Drug Administration (FDA) for the first-line therapy of advanced ALK-positive NSCLC patients [5].

The patient was kept on crizotinib as first-line therapy and showed a PFS of 48 months despite the data suggesting that most patients on crizotinib show relapse within 1 year [5].

Platinum-based double-agent chemotherapy has generally been the standard of care for the first-line and maintenance therapy of NSCLC except for an EGFR mutation-positive NSCLC. Moreover, for tumors with non-squamous histologic characteristics, cisplatin-pemetrexed is superior to cisplatingemcitabine [9]. In a prospective randomized phase III trial (Profile 1014) [10], crizotinib was compared with standard chemotherapy (pemetrexed plus platinum) in advanced ALKpositive NSCLC cases as first-line treatment and showed longer PFS of 10.9 months versus 7 months in the chemotherapy arm. These results were confirmed by another phase III randomized trial (Profile 1029) where crizotinib showed a significantly prolonged PFS of 11.1 months compared to 6.8 months with chemotherapy [11]. Taken together with these data, our patient showed considerably longer PFS of 48 months com- pared to 10.9 and 11.1 months reported by Profile 1014 and Profile 1029, respectively.

Several variants of the EML4-ALK fusion have been reported. To date, up to 15 variants have been identified. The most common variants are variant 1 , variant $3 \mathrm{a} / \mathrm{b}$, and variant 2 . Their expressions are $33 \%, 29 \%$ and $9 \%$, respectively [12]. The response to ALK TKI is heterogeneous and clinical outcomes in these patients vary. Some variants have been correlated with poor response to crizotinib due to acquired resistance, while other variants, as variant 2 suggested by Li et al, showed prolonged PFS with crizotinib [13]. However, many questions regarding the molecular basis for this association are yet to be answered. Unfortunately, we did not test for the $A L K$ variant here in our patient. Thus, we recommend testing for the $A L K$ variant to better recognize and predict treatment response.

Crizotinib has been known for its manageable and tolerable toxicity profile. Our patient presented with mild lower limb edema and grade I elevated transaminase level normalized after adding ursodeoxycholic acid without the need to stop the medication. This is in line with Profile 1014, where vision disorder (71\%), diarrhea (61\%), and edema (49\%) were the commonest adverse events [6]. While previous data have focused on different adverse events, our patient suffered from avascular necrosis that caused stress fracture and was pathologically proven. It is the second time to report such a rare finding with crizotinib intake after Takamori et al, who first reported rapidly destructive coxarthrosis in a 75-year-old female patient on crizotinib for 4.5 years [14]. Therefore, severe hip pain should not be neglected in those patients.

The magnitude of the improvement in PFS highly noticed in our case highlights the significance of determining the underlying factors of this robust response. Hence, additional research should focus on why some patients might not respond and the mechanisms behind resistance to help know why there is a difference in the duration of response among cases, hoping to optimize the treatment and outcomes.

\section{Conclusions}

We present an advanced ALK-positive NSCLC female patient who received crizotinib as first-line and maintenance therapy and showed PFS of 48 months, rarely reported with such medication. Also, she suffered from avascular necrosis of the left femur, which is a rare serious adverse event. Further studies should focus on the molecular and biological factors of this heterogeneous response, and future research should be made to better investigate avascular necrosis in those patients.

\section{Acknowledgments}

None to declare.

\section{Financial Disclosure}

None to declare. 


\section{Conflict of Interest}

None to declare.

\section{Informed Consent}

Informed consent was obtained from the patient.

\section{Author Contributions}

EB, ME, NO, MA, and MB all contributed to the case report's conception, writing, and editing.

\section{Data Availability}

The authors declare that data supporting the findings of this case study are available within the article.

\section{References}

1. Soda M, Choi YL, Enomoto M, Takada S, Yamashita Y, Ishikawa S, Fujiwara S, et al. Identification of the transforming EML4-ALK fusion gene in non-small-cell lung cancer. Nature. 2007;448(7153):561-566.

2. Li T, Maus MK, Desai SJ, Beckett LA, Stephens C, Huang E, Hsiang J, et al. Large-scale screening and molecular characterization of EML4-ALK fusion variants in archival non-small-cell lung cancer tumor specimens using quantitative reverse transcription polymerase chain reaction assays. J Thorac Oncol. 2014;9(1):18-25.

3. Peters S, Camidge DR, Shaw AT, Gadgeel S, Ahn JS, Kim DW, Ou SI, et al. Alectinib versus crizotinib in untreated ALK-positive non-small-cell lung cancer. N Engl J Med. 2017;377(9):829-838.

4. Christensen JG, Zou HY, Arango ME, Li Q, Lee JH, McDonnell SR, Yamazaki S, et al. Cytoreductive antitumor activity of PF-2341066, a novel inhibitor of anaplastic lymphoma kinase and c-Met, in experimental models of anaplastic large-cell lymphoma. Mol Cancer Ther. 2007;6(12 Pt 1):3314-3322.

5. Solomon BJ, Mok T, Kim DW, Wu YL, Nakagawa K, Mekhail T, Felip E, et al. First-line crizotinib versus chemotherapy in ALK-positive lung cancer. N Engl J Med. 2014;371(23):2167-2177.

6. Shaw AT, Kim DW, Nakagawa K, Seto T, Crino L, Ahn MJ, De Pas T, et al. Crizotinib versus chemotherapy in advanced ALK-positive lung cancer. N Engl J Med. 2013;368(25):2385-2394.

7. Jemal A, Siegel R, Xu J, Ward E. Cancer statistics, 2010. CA Cancer J Clin. 2010;60(5):277-300.

8. Jemal A, Bray F, Center MM, Ferlay J, Ward E, Forman D. Global cancer statistics. CA Cancer J Clin. 2011;61(2):69-90.

9. Scagliotti GV, Parikh P, von Pawel J, Biesma B, Vansteenkiste J, Manegold C, Serwatowski P, et al. Phase III study comparing cisplatin plus gemcitabine with cisplatin plus pemetrexed in chemotherapy-naive patients with advanced-stage non-small-cell lung cancer. J Clin Oncol. 2008;26(21):3543-3551.

10. Noonan SA, Camidge DR. PROFILE 1014: lessons for the new era of lung cancer clinical research. Transl Lung Cancer Res. 2015;4(5):642-648.

11. Wu YL, Lu S, Lu Y, Zhou J, Shi YK, Sriuranpong V, Ho JCM, et al. Results of PROFILE 1029, a phase III comparison of first-line crizotinib versus chemotherapy in East Asian patients with ALK-positive advanced non-small cell lung cancer. J Thorac Oncol. 2018;13(10):1539-1548.

12. Horn L, Pao W. EML4-ALK: honing in on a new target in non-small-cell lung cancer. J Clin Oncol. 2009;27(26):4232-4235.

13. Li Y, Zhang T, Zhang J, Li W, Yuan P, Xing P, Zhang Z, et al. Response to crizotinib in advanced ALK-rearranged non-small cell lung cancers with different ALK-fusion variants. Lung Cancer. 2018;118:128-133.

14. Takamori S, Seto T, Jinnouchi M, Oba T, Yamaguchi M, Takenoyama M. Rapidly destructive coxarthrosis as a potential side effect of crizotinib in a patient with ROS1positive lung adenocarcinoma. Ther Clin Risk Manag. 2020;16:17-20. 